\title{
Angelica Root Oil
}

National Cancer Institute

\section{Source}

National Cancer Institute. Angelica Root Oil. NCI Thesaurus. Code C107262.

The oil extracted from the roots of Ang elica archang elica. Angelica root oil is used in aromatherapy and to stimulate the immune system. 